\title{
Motor Axonal Regeneration following Cord Transection
}

\author{
Jason R. Plemel ${ }^{1,2 *}$ and Jacquelyn J. Cragg ${ }^{1,3 *}$ \\ ${ }^{1}$ International Collaboration on Repair Discoveries, ${ }^{2}$ Department of Neurosciences, and ${ }^{3}$ School of Population and Public Health, Faculty of Medicine, \\ University of British Columbia, Vancouver, British Columbia, Canada V5Z 1M9 \\ Review of Lu et al.
}

The goal of promoting regeneration of CNS fibers has baffled and excited neuroscientists for decades. Recent advances demonstrate the feasibility of promoting regeneration of single-fiber tracts by stimulating a growth response within neurons, altering the normally nonpermissive CNS environment, providing a favorable growth substrate to bridge the injury site, and/or producing a growth-promoting gradient (Alto et al., 2009; Kadoya et al., 2009; Sun et al., 2011). One contentious aspect in the field, however, is whether regeneration of damaged fibers is sufficient to restore function after CNS injury. Work by Lu and colleagues (2012) published in a recent issue of The Journal of Neuroscience yields evidence to suggest that more will be required to restore function after injury than simply promoting the regeneration of a given spinal tract.

Lu et al. (2012) used a rat lateral midcervical hemisection model and demonstrated that a combinatorial treatment strategy resulted in motor axon regeneration. This combinatorial strategy included administration of the cell-permeable dibutyryl cAMP in brainstem reticular motor nuclei, bone marrow stromal cell grafts

\footnotetext{
Received Aug. 13, 2012; revised Sept. 13, 2012; accepted Sept. 17, 2012. We thank Lesley Soril, Dr. Chris West, and Dr. Wolfram Tetzlaff for their insightful reviews of the manuscript.

The authors declare no competing financial interests.

*J.R.P. and J.J.C. contributed equally to this work.

Correspondence should be addressed to Jacquelyn J. Cragg, International Collaboration on Repair Discoveries, University of British Columbia, Blusson Spinal Cord Centre, 818 West 10th Avenue, Vancouver, BC, Canada V5Z 1M9. E-mail: craggj@interchange.ubc.ca.

DOI:10.1523/JNEUROSCI.3858-12.2012

Copyright $\odot 2012$ the authors $\quad 0270-6474 / 12 / 3215645-02 \$ 15.00 / 0$
}

in the lesion site, and brain-derived neurotrophic factor (BDNF) gradients beyond the lesion site. Despite the fact that this treatment promoted axonal regeneration in this group, all motor outcomes worsened, as assessed with grid walking, grooming, and gait analysis.

To remove the contribution of the intact reticulospinal tract, which is spared following lateral hemisection, Lu et al. (2012) also tested their optimal full combinatorial treatment in a rat upper thoracic full-transection model. Because the majority of motor assessments are inappropriate to use in fulltransection models, Lu et al. (2012) used open-field locomotion, assessed with a Beattie-Basso-Bresnahan (BBB) score. They demonstrated that $\mathrm{BBB}$ motor outcomes improved in animals receiving the same combinatorial treatment as used in the hemisection model. However, resection of regenerated fibers following full transection did not abolish gained function, suggesting that the regeneration observed was not responsible for the improvement in motor function.

One possible explanation for why resection did not abolish function is that the treatment resulted in hyperexcitability of spinal circuitry. The authors explored this possibility by using a five-point spasticity scale in response to a standardized stretch/ rub maneuver and found that BDNF treatment below the transection site was associated with heightened spasticity. Thus, the combined therapeutic strategy promoted regeneration in both lesion models and was associated with either worsened motor function or enhanced spasticity.

What else, besides axonal regeneration, might be required to restore motor function? Lu et al. (2012) investigated and ruled out several possibilities. First, a sufficient number of axons must regrow. $\mathrm{Lu}$ and colleagues (2012) reported that $\sim 400$ bridging axons regenerated $500 \mu \mathrm{m}$ caudal to the graft. This might be sufficient to improve motor function, because the regeneration and reentry of $\sim 300$ axons across a peripheral nerve bridge increase forelimb range of motion after a cervical hemisection (Houle et al., 2006). Second, regenerative fibers might require remyelination to sustain axonal conduction and thus restore function, given that demyelination can block the conduction of action potentials (Waxman, 1977) as a result of both the paucity of sodium channels on the axolemma of the demyelinated internode and the high number of potassium channels in the adjacent juxtaparanode (Waxman, 2006). Lu and colleagues (2012) demonstrated that $81 \%$ of regenerated fibers in the white matter were surrounded by myelin proteins such as myelin basic protein and myelin oligodendrocyte glycoprotein, suggesting that these regenerated fibers were indeed remyelinated. Third, axons must make functional and stable synapses to mediate synaptic transmission and reestablish function. Using confocal and electron microscopy, Lu et al. (2012) found that regenerated axons associated with synaptic markers and displayed structural characteristics of synapse formation. One major 
limitation of these techniques, however, is that it is not possible to determine whether these synaptic structures are functional. Electrophysiological techniques, routinely used to assess synaptic activity (Ramer et al., 2000; Bonner et al., 2011) would have been a superior assay of functional synapse formation. Previous findings suggest that the regeneration of dorsal column axons, for example, fails to produce a detectable electrophysiological response in the presence of readily detected synapses (Alto et al., 2009). With such available techniques, it would be possible to validate the structural findings in the full transection model.

It is important to note that even if some axon tracts make appropriate functional synapses with their normal targets, all functions will not necessarily recover; the appropriate tracts must regenerate. As previously mentioned, $\mathrm{Lu}$ and colleagues (2012) found that the regeneration of reticulospinal fibers was not associated with improvement in forelimb function in the hemisection model, as assessed by a gridwalking task or a grooming test. However, the hemisection model also severs rubrospinal and corticospinal tracts that control paw and forelimb use in arpeggio and grasping (Whishaw et al., 1998). The combinatorial strategy targeted regeneration only of the reticulospinal tract; corticospinal and rubrospinal regeneration were not assessed. While the authors used several appropriate behavioral tests that assess a range of functions for this injury model, it is possible that regeneration of the reticulospinal tract alone may not be sufficient to restore forelimb functions. It would be important, looking forward, to determine whether there is greater potential for certain motor tracts to mediate functional recovery by using combinatorial strategies to promote the growth of other motor tracts.

In testing regenerative therapies, strategies designed to improve motor function can also promote the plasticity of unintended targets, with potentially harmful consequences. The resultant combination treatment-induced increase in spasticity could potentially mask the beneficial effects of axonal regeneration. Spasticity might have resulted from BDNF expres- sion, both as a result of the injury itself, and from the combinatorial treatment, which included BDNF-expressing cell grafts in the lesion site and BDNF gradients beyond the lesion site. One mechanistic candidate relating BDNF and spasticity is the expression of the potassium-chloride cotransporter-2 (KCC2) in ventral horn motor neurons. Normally, KCC2 regulates chloride gradients by keeping the intracellular concentrations of chloride ions low. Spinal cord injury (SCI) results in a BDNFdependent downregulation of KCC2, which results in a more positive equilibrium potential of chloride (Boulenguez et al., 2010). This converts the inhibitory effect of GABA to an excitatory one, thus increasing motor neuron activity and enhancing spasticity.

The beneficial effects of axonal regeneration may have also been masked by neuropathic pain. It has been demonstrated that peripheral nerve injury stimulates BDNF release from microglia, which induces downregulation of KCC2 in spinal lamina I neurons, resulting in mechanical allodynia (Coull et al., 2005). As intrathecal delivery of BDNF is also sufficient to induce tactile allodynia (Coull et al., 2005), the BDNF gradients beyond the lesion site are predicted to produce symptoms of neuropathic pain. It may have therefore been telling for Lu et al. (2012) to examine pain behavior in addition to motor functions and spasticity in their animal model. From a clinical perspective, relieving pain is a high priority in individuals with SCI, and is frequently overlooked in outcome assessments for regenerative treatment strategies.

Overall, Lu et al. (2012) provide an important contribution to the field of neuroscience in demonstrating axonal regeneration following partial and complete cord transection. Perhaps surprisingly, this regeneration of a descending motor tract is not associated with improvements in motor function, highlighting the complexity of axonal regeneration and how it relates to restoring motor function. Given that there are no current therapeutic interventions for treatment of SCI, there is an exigent need to further investigate regenerative therapies and assess how regeneration relates to functional recovery.

\section{References}

Alto LT, Havton LA, Conner JM, Hollis ER 2nd, Blesch A, Tuszynski MH (2009) Chemotropic guidance facilitates axonal regeneration and synapse formation after spinal cord injury. Nat Neurosci 12:1106-1113.

Bonner JF, Connors TM, Silverman WF, Kowalski DP, Lemay MA, Fischer I (2011) Grafted neural progenitors integrate and restore synaptic connectivity across the injured spinal cord. J Neurosci 31:4675-4686.

Boulenguez P, Liabeuf S, Bos R, Bras H, JeanXavier C, Brocard C, Stil A, Darbon P, Cattaert D, Delpire E, Marsala M, Vinay L (2010) Down-regulation of the potassium-chloride cotransporter KCC2 contributes to spasticity after spinal cord injury. Nat Med 16:302-307.

Coull JA, Beggs S, Boudreau D, Boivin D, Tsuda M, Inoue K, Gravel C, Salter MW, De Koninck $Y$ (2005) BDNF from microglia causes the shift in neuronal anion gradient underlying neuropathic pain. Nature 438:1017-1021.

Houle JD, Tom VJ, Mayes D, Wagoner G, Phillips N, Silver J (2006) Combining an autologous peripheral nervous system "bridge" and matrix modification by chondroitinase allows robust, functional regeneration beyond a hemisection lesion of the adult rat spinal cord. J Neurosci 26:7405-7415.

Kadoya K, Tsukada S, Lu P, Coppola G, Geschwind D, Filbin MT, Blesch A, Tuszynski MH (2009) Combined intrinsic and extrinsic neuronal mechanisms facilitate bridging axonal regeneration one year after spinal cord injury. Neuron 64:165-172.

Lu P, Blesch A, Graham L, Wang Y, Samara R, Banos K, Haringer V, Havton L, Weishaupt N, Bennett D, Fouad K, Tuszynski MH (2012) Motor axonal regeneration after partial and complete spinal cord transection. J Neurosci 32:8208-8218.

Ramer MS, Priestley JV, McMahon SB (2000) Functional regeneration of sensory axons into the adult spinal cord. Nature 403:312-316.

Sun F, Park KK, Belin S, Wang D, Lu T, Chen G, Zhang K, Yeung C, Feng G, Yankner BA, He Z (2011) Sustained axon regeneration induced by co-deletion of PTEN and SOCS3. Nature 480:372-375.

Waxman SG (1977) Conduction in myelinated, unmyelinated, and demyelinated fibers. Arch Neurol 34:585-589.

Waxman SG (2006) Axonal conduction and injury in multiple sclerosis: the role of sodium channels. Nat Rev Neurosci 7:932-941.

Whishaw IQ, Gorny B, Sarna J (1998) Paw and limb use in skilled and spontaneous reaching after pyramidal tract, red nucleus and combined lesions in the rat: behavioral and anatomical dissociations. Behav Brain Res 93: 167-183. 